\title{
A Thermodynamic Model for Multivalency in 14-3-3 Protein-Protein Interactions
}

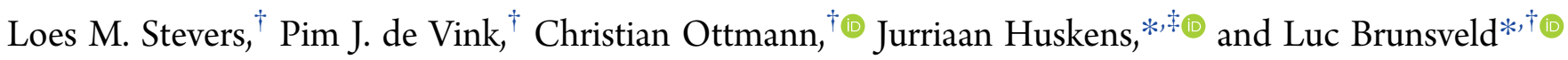 \\ ${ }^{\dagger}$ Laboratory of Chemical Biology, Department of Biomedical Engineering and Institute for Complex Molecular Systems, Technische \\ Universiteit Eindhoven, P.O. Box 513, Eindhoven 5600 MB, The Netherlands \\ ${ }^{\ddagger}$ Molecular NanoFabrication Group, MESA+ Institute for Nanotechnology, University of Twente, P.O. Box 217, Enschede 7500 AE, \\ The Netherlands
}

\section{Supporting Information}

\begin{abstract}
Protein-protein interactions (PPIs) are at the core of molecular control over cellular function. Multivalency in PPI formation, such as via proteins with multiple binding sites and different valencies, requires fundamental understanding to address correlated challenges in pathologies and drug development. Thermodynamic binding models are needed to provide frameworks for describing multivalent PPIs. We established a model based on ditopic host-guest systems featuring the effective molarity, a hallmark property of multivalency, as a prime parameter governing the intramolecular binding in divalent interactions. By way of illustration, we study the interaction of the bivalent 14-3-3

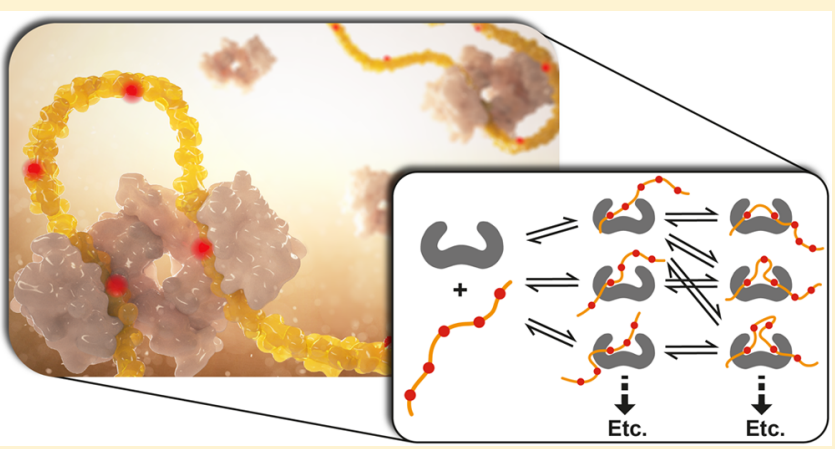
protein scaffold with both the nonavalent CFTR and the hexavalent LRRK2 proteins, determining the underlying thermodynamics and providing insights into the role of individual sites in the context of the multivalent platform. Fitting of binding data reveals enthalpy-entropy correlation in both systems. Simulations of speciations for the entire phosphorylated protein domains reveal that the CFTR protein preferably binds to 14-33 by combinations including the strongest binding site pS768, but that other binding sites take over when this site is eliminated, leading to only a minor decrease in total affinity for 14-3-3. For LRRK2, two binding sites dominate the complex formation with 14-3-3, but the distantly located pS1444 site also plays a role in complex formation. Thermodynamic modeling of these multivalent PPIs allowed analyzing and predicting the effects of individual sites regarding their modulation via, for example, (de)phosphorylation or small-molecule targeting. The results specifically bring forward the potential of PPI stabilization, as an entry for drug discovery for multivalent PPIs.
\end{abstract}

\section{INTRODUCTION}

Multivalency involves the combination of several individual bonds or binding events and is a common feature in the regulation of biological systems. ${ }^{1}$ By combining several, typically isolated, interaction sites, strong yet reversible binding can be achieved between two partners, even when the individual interactions are relatively weak. ${ }^{2}$ Next to this, multivalency can lead to ultrasensitivity in signal regulation, which is important for rapid, nonlinear, and low-noise transitions in biological systems. ${ }^{3}$ Multivalency thus provides control over the signal transduction mediated by supramolecular assemblies of proteins. Multivalency in biological systems has been studied in detail for biomolecular systems like carbohydrate-lectin interactions and divalent antibodies. ${ }^{1,2}$ However, the fundamental aspects of multivalency in intracellular regulation mechanisms such as protein-protein interactions (PPIs) have seen much less attention, possibly because of their very diverse nature and lack of easily accessible model systems. PPIs are at the core of molecular control over cellular function ${ }^{4,5}$ and therefore constitute a valid therapeutic entry via either their inhibition or stabilization. ${ }^{6-8}$ The focus in PPI modulation has typically been on one-to-one complexes with rather well-defined, monovalent interaction sites. PPI complexes formed via multivalent interaction sites are still very much unchartered space, in part because of the underlying complexity, with resulting challenges such as achieving selectivity, affinity, and functional regulation not being addressed.

Almost all proteins are built up from multiple domains. ${ }^{9}$ As a result, multidomain or multisite interactions are prominent features in PPI regulation. ${ }^{10,11}$ Scaffolding is one example of how multiple domains can act in synergy for efficient interplay of protein signaling and ultrasensitivity. ${ }^{12,13}$ Next to that, multidomain interactions are in general a powerful method to control affinity and selectivity in PPIs. ${ }^{14-21}$ Intrinsically disordered protein elements, capable of adjusting to different spacings/distances between multiple domains and befitted with

Received: September 5, 2018

Published: October 8, 2018 
A

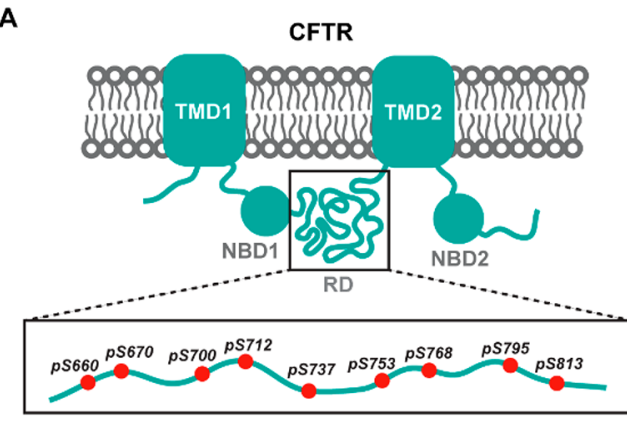

C

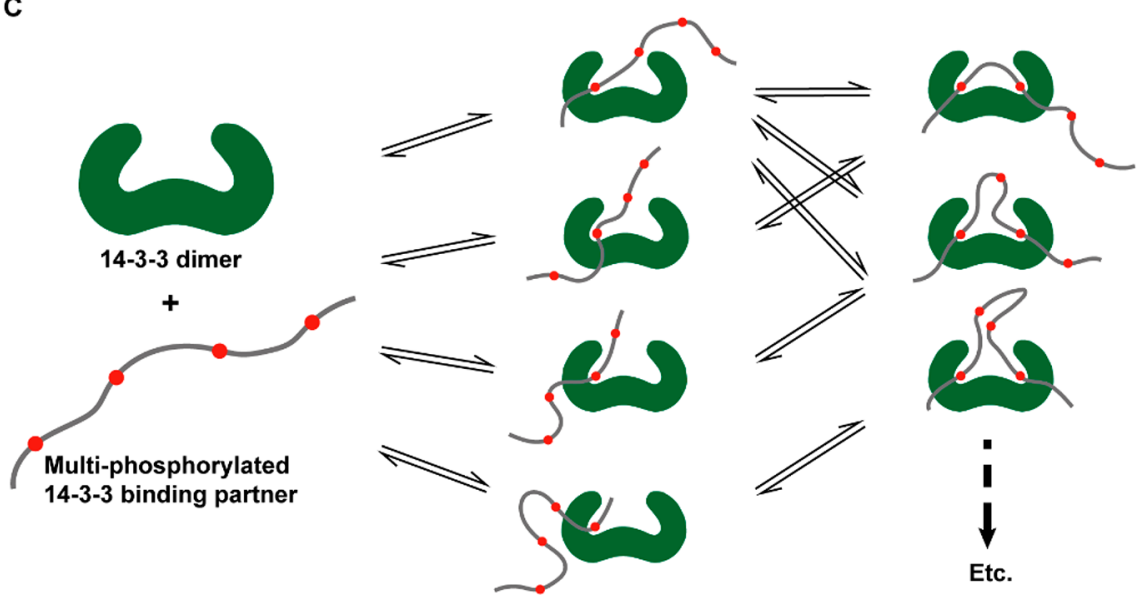

B

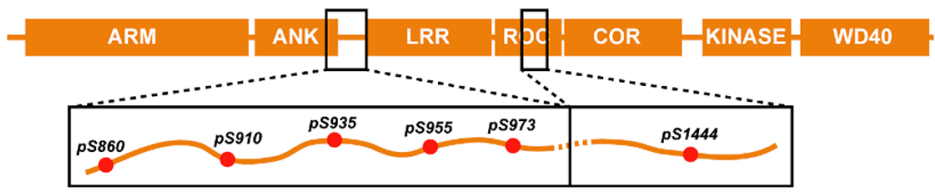

Figure 1. Multivalency in 14-3-3 protein-protein interactions. Overview of (A) the nine phosphorylated 14-3-3 interaction sites within the intrinsically disordered Regulatory Domain (RD) of CFTR and (B) the six phosphorylated 14-3-3 interaction sites within either the intrinsically disordered region between the ANK and LRR domain or the ROC domain of LRRK2. (C) Schematic representation of different binding modes of a multivalent 14-3-3 binding partner to the dimeric 14-3-3 scaffold, via either monovalent or bivalent binding.

a high flexibility, play an important role in enabling these multidomain interactions. ${ }^{22-27}$ Multivalent interactions between protein domains beyond the di- and trivalent type can even be responsible for local phase separations in the cellular environment. ${ }^{28-30}$ The implications of discontinuous multivalent interactions in PPI regulation (beyond the "hot regions") for drug discovery have not received substantial attention. ${ }^{6}$ The discontinuity of the binding sites, either on one or on multiple protein domains (Figure 1), is a significant challenge for the development of small molecules targeting this interaction. ${ }^{31-34} \mathrm{~A}$ fundamental, thermodynamic understanding of the resulting character of PPIs with separate multivalent and noncontiguous hot-spot constellations is thus needed.

14-3-3 proteins are a family of seven functionally dimeric scaffold proteins that interact with a plethora of signaling proteins and that are involved in many cellular process and correlated diseases. ${ }^{35}$ As a result, 14-3-3 proteins have been studied as PPI-based drug targets. ${ }^{36}$ The 14-3-3 adapter proteins themselves are stably folded dimers, but their severalhundred protein partners and interacting elements, which typically bind through short phosphorylated motifs, are frequently disordered..$^{37} \mathrm{~A}$ significant portion of the interaction partners of 14-3-3 proteins contain multiple phosphorylated binding epitopes, thus effectively forming multivalent PPIs. Cystic Fibrosis Transmembrane conductance Regulator $(\text { CFTR })^{38}$ and Leucine-Rich Repeat Kinase 2 (LRRK2) ${ }^{39}$ are two important and disease-related proteins, of which the activity is regulated via their interaction with 14-3-3 proteins, and they feature multiple (nine and six, respectively) phosphorylated binding sites for the binary 14-3-3 platform.
The binding of such multiphosphorylated proteins to the bivalent 14-3-3 platform is typically heterotopic and occurs via heterogeneous spacings, two features basically not touched upon in current conceptual approaches toward multivalent PPIs. ${ }^{10,11}$

Here, we provide detailed fundamental analyses of these two multivalent PPI systems. Different modes of multivalent interaction are analyzed and compared regarding their thermodynamic characteristics. For this, multivalent thermodynamic binding models were devised to provide a general framework for classifying the individual and multivalent interactions and to obtain values for the effective molarity (EM), a property that is the hallmark of multivalency. The thermodynamic model was then used to simulate the binding of full protein constructs to 14-3-3 and to provide insights into the role of individual sites in the context of the multivalent platform as well as resulting effects of their modulation, for example, via (de)phosphorylation or small-molecule stabilization.

\section{RESULTS}

Mutations in the CFTR protein frequently result in defective intracellular transport and processing, and are critically correlated with the occurrence of cystic fibrosis $(\mathrm{CF}) .^{40}$ The binding of 14-3-3 proteins to the disordered Regulatory Domain (RD) of CFTR enhances trafficking of this chloride channel to the plasma membrane. ${ }^{41}$ The $\mathrm{RD}$ features nine chemically distinct 14-3-3 binding sites with which it can bind via a number of different binding constellations to the 14-3-3 dimer (Figure 1A)..$^{38,42}$ These numerous heterogeneous 
multivalent PPI possibilities (Figure 1C) make the interaction between CFTR and 14-3-3 highly attractive for fundamental studies of the underlying thermodynamics, especially because individual binding data of individual sites and diverse bivalent combinations can be determined. ${ }^{38}$ Similarly, the LRRK2 protein, which can be impaired by a number of mutations relevant for Parkinson's Disease (PD), is known to bind to 143-3 via six phosphorylated potential binding sites. ${ }^{43}$ Interestingly, the LRRK2 protein has five 14-3-3 binding sites in close proximity, located in a disordered region between the ankyrin (ANK) and leucine-rich repeats (LRR) domains, and an additional site located in the more distant Ras of complex proteins (ROC) domain (Figure 1B). Combinations of two phosphorylated LRRK2 sites enhance the binding affinity for 14-3-3 significantly. ${ }^{39}$ The combinations of both protein complexes that are ultimately required for optimal biological regulation, the interplay of the different multivalent PPIs possible, and the implications for biomedical intervention remain however unclear.

A Heterodivalent Noncooperative Binding Model. We used a molecular host-guest description to develop a model that describes the interactions of 14-3-3 with phosphorylated peptides. In this model, the 14-3-3 dimer is considered a ditopic host with two identical binding pockets. ${ }^{44}$ This host molecule can accommodate either two singly phosphorylated peptides, which can be described as monotopic guest molecules (Figure S2), but also one doubly phosphorylated peptide, which can be described as a ditopic guest molecule and requires to take the concept of multivalency into account (Figure S3). Furthermore, the CFTR and LRRK2 proteins feature different amino acid sequences flanking the phosphoserine epitopes, which makes the system heteroditopic (Figure 2 ). Figure $2 \mathrm{~A}$ shows that such a multivalent binding can occur via two pathways: first the binding of one binding site or first the binding of the other, followed by the binding of the remaining binding site. In both cases, the second binding event cannot be compared directly to the intrinsic affinity constant, and the effective molarity term (EM) is needed to describe the

$$
\begin{aligned}
& \text { A }
\end{aligned}
$$

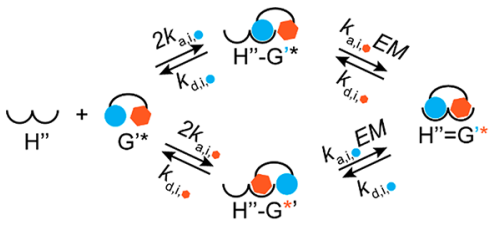

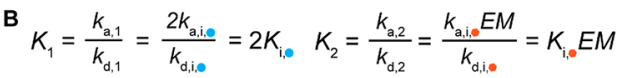

$$
\begin{aligned}
& K_{3}=\frac{k_{\mathrm{a}, 3}}{k_{\mathrm{d}, 3}}=\frac{2 k_{\mathrm{a}, \Theta}}{k_{\mathrm{d}, \Theta}}=2 K_{\mathrm{i}, \Theta} \quad K_{4}=\frac{k_{\mathrm{a}, 4}}{k_{\mathrm{d}, 4}}=\frac{k_{\mathrm{a}, \mathrm{e},} E M}{k_{\mathrm{di}, \Theta}}=K_{\mathrm{i}, \Theta} E M \\
& {\left[H^{\prime \prime}\right]_{\text {tot }}=\left[H^{\prime \prime}\right]+\left[H^{\prime \prime}-G^{\prime *}\right]+\left[H^{\prime \prime}-G^{* \prime}\right]+\left[H^{\prime \prime}=G^{\prime *}\right]} \\
& {\left[G^{\prime *}\right]_{\text {tot }}=\left[G^{\prime *}\right]+\left[H^{\prime \prime}-G^{\prime *}\right]+\left[H^{\prime \prime}-G^{\star \prime}\right]+\left[H^{\prime \prime}=G^{\prime *}\right]} \\
& K_{1}=\frac{\left[H^{\prime \prime}-G^{\prime *}\right]}{\left[H^{\prime \prime}\right]\left[G^{\prime *}\right]} K_{2}=\frac{\left[H^{\prime \prime}=G^{\prime *}\right]}{\left[H^{\prime \prime}-G^{* *}\right]} K_{3}=\frac{\left[H^{\prime \prime}-G^{* \prime}\right]}{\left[H^{\prime \prime}\right]\left[G^{\prime *}\right]} K_{4}=\frac{\left[H^{\prime \prime}=G^{\prime *}\right]}{\left[H^{\prime \prime}-G^{* \prime}\right]} \\
& K_{\text {ov }}=\frac{\left[H^{\prime \prime}=\mathrm{G}^{\prime *}\right]}{\left[\mathrm{H}^{\prime \prime}\right]\left[\mathrm{G}^{\prime *}\right]}=K_{1} K_{2}=K_{3} K_{4}=2 K_{\mathrm{i}, 9} K_{\mathrm{i}, \mathrm{e}} E M
\end{aligned}
$$

Figure 2. Model description of a heterodivalent, noncooperative 1:1 host-guest system. (A) The equilibrium between the 14-3-3 ditopic host molecule $\mathrm{H}^{\prime \prime}$ and heteroditopic guest molecule $G^{\prime *}$, monovalent complex $\mathrm{H}^{\prime \prime}-\mathrm{G}^{*}$, monovalent complex $\mathrm{H}^{\prime \prime}-\mathrm{G}^{* \prime}$, and divalent complex $\mathrm{H}^{\prime \prime}=G^{\prime *}$. (B) Equilibrium and mass balance equations for the interaction. overall binding affinity. The EM depends on the length and flexibility of the linker between the two different binding sites, and is closely related to the effective concentration $\left(C_{\text {eff }}\right)$ : the probability that the two reactive groups find each other in the restricted volume imposed by the molecular connection between the sites. ${ }^{45}$ The overall affinity constant (Kov) is thus critically dependent on three parameters: the two affinity constants of the individual motifs and the EM (Figure 2B).

Fluorescence polarization (FP) and isothermal titration calorimetry (ITC) experiments were performed to quantitatively analyze the binding between 14-3-3 and singly and doubly phosphorylated CFTR and LRRK2 peptides (see Tables S1 and S2 for the amino acid sequences and Figures S6-S12 for the experimental data). The selection of the isoform 14-3-3 $\beta$ for CFTR and 14-3-3 $\gamma$ for LRRK2 relates to their physiological relevance in cellular systems. ${ }^{38,39}$ FP assays are relatively high throughput and highly sensitive, which allows the use of low concentrations, suited to assess strong interactions. ITC experiments consume relatively high amounts of material and have lower sensitivity, but provide direct access to complexation enthalpies and are therefore able to yield the complete thermodynamic picture, including enthalpic and entropic contributions to the interaction, in a single titration experiment. The acquired data were fitted on the basis of the models given in Figures 2 and S2, eqs S1-S3, and the corresponding clarification in the Supporting Information. All fits and resulting $K_{\mathrm{d}}$ 's and thermodynamic parameters $\Delta H$ and $-T \Delta S$ are displayed and provided in Figures S5-S11 and Tables S3 and S4. A notable observation includes the strong affinities of most of the singly phosphorylated LRRK2 peptides in comparison to the singly phosphorylated CFTR peptides. Consequently, the FP assays typically could not provide the full binding curve for the weaker binding singly phosphorylated CFTR peptides. In case of the doubly phosphorylated peptides, the very high affinities (nM) of some LRRK2 sequences were pushing the detection limits, while the affinities of the doubly phosphorylated CFTR peptides were now reaching physiologically relevant values, easy determinable using both FP and ITC.

Enthalpy-Entropy Correlation and Effective Molarity. The binding enthalpies of the ditopic doubly phosphorylated peptides to 14-3-3 are compared to the binding enthalpies of the singly phosphorylated peptides and their sum (Table 1). This comparison is only possible for the LRRK2 peptides as the binding of the singly phosphorylated CFTR peptides was too low to obtain reliable binding enthalpies. For four of the LRRK2 peptides, ITC data of both the doubly and the singly phosphorylated peptides are available. Interestingly, for the values of three of these peptides, the difference between $\Delta H$ of the doubly phosphorylated peptide and the sum of the $\Delta H$ values of the singly phosphorylated peptides is small $(<1 \mathrm{kcal} / \mathrm{mol})$, probably within experimental error, indicating little to no participation of the linker in the binding to 14-3-3. Only for peptide LRRK2_pS910pS935 is the difference in binding enthalpy between the divalent peptide and the sum of the monovalent peptides LRRK2_pS910 and LRRK2_pS935 significantly larger $(4.6 \mathrm{kcal} / \mathrm{mol})$ with a more exothermic binding of the divalent peptide. Notably, the independent behavior of the pS910 and pS935 sites in other doubly phosphorylated peptides is confirmed by proper additivity, thus underlining the special nature of the LRRK2_pS910pS935 peptide. Below it will be shown that also the EM of this ditopic peptide is 
Table 1. Comparison between the Binding Enthalpies of the Doubly Phosphorylated Peptides and the Sum of the Two Corresponding Singly Phosphorylated Peptides Binding to 14-3-3 ${ }^{a}$

$\begin{array}{lccccc} & \Delta H \text { mono } 1(\mathrm{kcal} / \mathrm{mol}) & \Delta H \text { mono } 2(\mathrm{kcal} / \mathrm{mol}) & \text { sum } \Delta H \text { mono }(\mathrm{kcal} / \mathrm{mol}) & \Delta H \text { ditopic }(\mathrm{kcal} / \mathrm{mol}) & \text { difference }(\mathrm{kcal} / \mathrm{mol}) \\ \text { CFTR_pS753pS768 } & -8.7 & -2.3 & \mathrm{X} & -11.0 \\ \text { CFTR_pS768pS795 } & -2.3 & -12.5 & \mathrm{X} & -14.8 \\ \text { CFTR_pS795pS813 } & -12.5 & -7.5 & \mathrm{X} & -20.0 & -12.3 \\ \text { CFTR_pS712pS768 } & -10.0 & -2.3 & \mathrm{X} & -9.8 \\ \text { CFTR_pS768pS813 } & -2.3 & -7.5 & \mathrm{X} & -11.9 & \\ \text { LRRK2_pS860pS910 } & -7.1 & -4.9 & -12.5 & -17.1 & \\ \text { LRRK2_pS910pS935 } & -4.9 & -7.6 & -13.7 & -14.5 & 0.6 \\ \text { LRRK2_pS935pS955 } & -7.6 & -6.1 & \mathrm{X} & -6.6 & 0.8 \\ \text { LRRK2_pS955pS973 } & -6.1 & -0.5 & -14.8 & -15.7 & 0.9 \\ \text { LRRK2_pS910pS1444 } & -4.9 & -10.0 & -17.6 & -17.6 & 0.0\end{array}$

${ }^{a}$ Measured values are shown in roman type, and values calculated from additivity are shown in italic. Experiments that have not been performed are noted as "X".
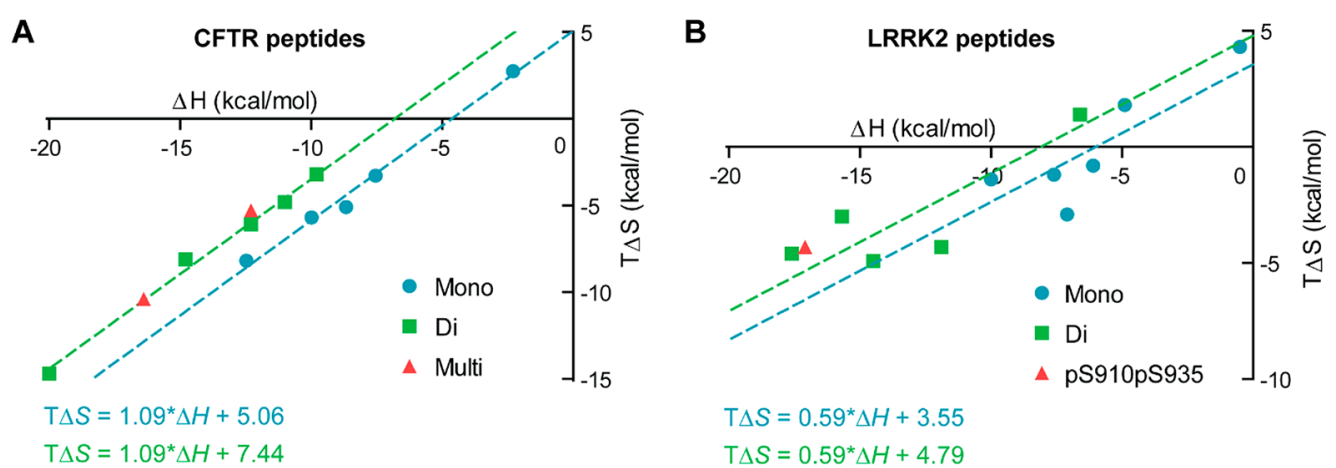

Figure 3. Enthalpy $(\Delta H)$-entropy $(T \Delta S)$ correlation plots of (A) CFTR peptides binding to 14-3-3 $\beta$ and (B) LRRK2 peptides binding to 14-3$3 \gamma$. The data points of the singly phosphorylated peptides are plotted in blue, and the doubly phosphorylated peptides are in green. The formulas of the trend lines fitted through these data points are shown below the graphs in the corresponding color. Red data points represent the triply phosphorylated CFTR_pS753pS768pS795 peptide, the full R-domain, and peptide LRRK2_pS910pS935, and are not used in the trend line fits.

higher than expected for independent binding and higher than that of the other ditopic peptides, and it will be explained that this behavior is in agreement with the crystal structure, which indicates a strong involvement of the linker for this ditopic peptide only. When assuming that for all other ditopic peptides the role of the linker in the binding is low, the binding enthalpies of a number of singly phosphorylated peptides can be calculated from additivity on the basis of the enthalpy of the doubly phosphorylated peptides with overlapping sequences (in italic in Table 1). For the LRRK2 system, this has been done for the pS860 and pS973 sites only, while for the CFTR system all monovalent binding enthalpies given in Table 1 have been calculated in this manner.

The binding enthalpies can be used to calculate the entropy values of binding of these peptides to 14-3-3 using eq S3 and the $K_{\mathrm{d}}$ values measured with FP from Tables S3 and S4. Figure 3 shows the correlation between the enthalpy and entropy of the singly phosphorylated (blue 0 ) and doubly phosphorylated peptides (green $\square$ ) of both CFTR and LRRK2 binding to the two isoforms 14-3-3 $\beta$ and 14-3-3 $\gamma$, respectively. Both the singly and the doubly phosphorylated CFTR peptides binding to $14-3-3 \beta$ visibly reveal a strong, linear correlation between $\Delta H$ and $T \Delta S$, with a similar slope but different intercepts. Actually, when fitting the data for the singly and doubly phosphorylated CFTR peptides separately, very similar slopes of 1.08 and 1.10, respectively, were obtained (fits not shown). The difference in intercepts between the singly and doubly phosphorylated peptides can be explained on the basis of the equations in Figure $2\left(K_{\mathrm{di}}=2 K_{\mathrm{mono}, 1} K_{\mathrm{mono}, 2} \mathrm{EM}\right)$ and eq S3. When the trend line of the singly phosphorylated peptides is written as eq 1 , then the trend line of the doubly phosphorylated peptides can be written as eq 2 , in which $a$ and $b$ indicate the slope and intercept, respectively, of the linear functions.

$$
\begin{aligned}
& T \Delta S_{\text {mono }}=a^{*} \Delta H_{\text {mono }}+b \\
& T \Delta S_{\mathrm{di}}=a^{*} \Delta H_{\mathrm{di}}+2 b+R T \ln \left(2 \mathrm{EM}_{\mathrm{ov}}\right)
\end{aligned}
$$

All data points for both the mono- and the ditopic peptides can thus be fitted with only three parameters, $a$ (which is the slope that now fits both the singly and the doubly phosphorylated peptides together), $b$, and the overall effective molarity $\mathrm{EM}_{\mathrm{ov}}$. This provides a more strict and more informative model than fitting the mono- and ditopic series independently (requiring four parameters). With this model, for the CFTR peptides, a slope $(a)$ of 1.09 was obtained, which is in excellent agreement with the values of the slopes of the separately fitted sets as described above. A triply phosphorylated peptide and full R-domain (vide infra) were left out of the calculations, but plotted afterward in the graphs as red $\mathbf{\Lambda}$. Notably, the data for these two molecules fit nicely on the trend line of the doubly phosphorylated peptides, which is attributed to two binding sites that can bind in the grooves of a 14-3-3 dimer, while higher binding valencies are considered physically impossible. The enthalpy-entropy correlation of the LRRK2 peptides contains more noise, but the same theory can 
be applied. The LRRK2 pS910pS935 was left out of the calculations but plotted afterward in the graph with a red $\mathbf{\Delta}$. This peptide, however, seems to fit on the enthalpy-entropy correlation line of the doubly phosphorylated LRRK2 peptides as well. This clear correlation between the enthalpy and entropy can possibly be explained by the number of amino acid residues participating in the binding to 14-3-3: more amino acid residues binding in the groove will be favorable for the enthalpy, but the conformational restrictions the peptide has to make to bind will be unfavorable entropically.

Enthalpy-entropy correlations are often linear. ${ }^{46,47}$ This effect is also called enthalpy-entropy compensation, because a positive slope observed for a series of structurally similar complexes indicates that a tighter binding, visible by a stronger exothermic enthalpy, is associated with a more unfavorable binding entropy, resulting in a reduced effect on the binding free energy. The slope of the enthalpy-entropy graph is therefore a sign of how strong a change in enthalpy is translated into a change of the entropy. A clear difference is here observed in the slope of the trend lines for CFTR and LRRK2. It is interesting to note that the slope for the CFTR system is close to 1 , which means that any change in binding enthalpy is fully compensated by a change in entropy. This results in an approximately zero change in the binding free energy, thus explaining the observed very similar $K_{d}$ values found for this system (Table S3). In more detail, the slope is slightly higher than 1 (i.e., 1.09), which indicates that the strongest binders are actually entropy-driven, and that ligands with a more favorable binding enthalpy bind (slightly) less strongly. In contrast, the compensation effect is much smaller for LRRK2, with a slope of below 1 (0.59), which indicates that (i) the spread in $K_{\mathrm{d}}$ values (Table S4) is a lot larger than for CFTR, and (ii) the strongest binders are the ligands with the most favorable binding enthalpies.

The three-parameter fit, using $a, b$, and $\mathrm{EM}_{\mathrm{ov}}$ as shown in eqs 1 and 2 , of the $T \Delta S$ versus $\Delta H$ graphs for CFTR and LRRK2 yields the overall effective molarities $\mathrm{EM}_{\text {ov }}$, basically resulting from the difference in intercept of the mono- versus divalent peptides. These $\mathrm{EM}_{\mathrm{ov}}$ values are based on the rough approximation that the EMs are equal for all divalent peptides. For the CFTR system, the calculated $\mathrm{EM}_{\text {ov }}$ was $5.5 \mathrm{mM}$, while for LRRK2 a value of $10.2 \mathrm{mM}$ was obtained. The good agreement between these values corresponds with the structural similarity of the two 14-3-3 proteins used in this research. On the basis of the model depicted in Figure 2, the individual EM values of the doubly phosphorylated peptides can be calculated as well, from their $K_{\mathrm{d}}$ values and the $K_{\mathrm{d}}$ values of the singly phosphorylated peptides (Table 1 ), using the FP binding data. With an exception of peptide LRRK2 pS910pS935, the calculated EM values are all between 1 and $26 \mathrm{mM}$ (Table 2). These values agree well with the overall $\mathrm{EM}_{\mathrm{ov}}$ values obtained from the enthalpyentropy plots. To correlate these values to structure, an effective concentration of $1-26 \mathrm{mM}$ can be converted to a distance between unbound host site and the free guest site of a sphere of $24.8 \AA(26 \mathrm{mM})$ to $73.5 \AA(1 \mathrm{mM}),{ }^{45}$ which agrees with the theoretical molecular distances in 14-3-3 crystallography (see Figure S13).

The effect of the "linker length" on the EM was investigated by plotting the calculated individual EM values as a function of the number of amino acids between the two phosphorylated serine residues in the peptides (Figure S14). No significant trend was found between these two parameters. However,
Table 2. Effective Molarities of the Doubly Phosphorylated CFTR and LRRK2 Peptides As Compared To the Distance between the Phosphorylated Serine Residues in Number of Amino Acids

\begin{tabular}{lcc}
\hline \multicolumn{1}{c}{ peptide } & EM (mM) & distance (no. aa) \\
CFTR_pS660pS670 & 3.5 & 9 \\
CFTR_pS670pS700 & 14 & 29 \\
CFTR_pS700pS712 & 5.8 & 11 \\
CFTR_pS712pS737 & 1.7 & 24 \\
CFTR_pS737pS753 & 6.1 & 15 \\
CFTR_pS753pS768 & 20 & 14 \\
CFTR_pS768pS795 & 25 & 26 \\
CFTR_pS795pS813 & 6.8 & 17 \\
LRRK2_pS860pS910 & 2.5 & 19 \\
LRRK2_pS910pS935 & 110 & 24 \\
LRRK2_pS935pS955 & 21 & 19 \\
LRRK2_pS955pS973 & 26 & 17 \\
LRRK2_pS910pS1444 & 1.2 & 19 \\
LRRK2_pS935pS1444 & 1.9 & 19 \\
\hline
\end{tabular}

because 14-3-3 crystal structures show that the number of amino acid residues participating in the interaction with the 14-3-3 binding groove is different for each binding site, ${ }^{38,39}$ it is therefore difficult to say how long the actual flexible element of the linker is and which amino acid residues are part of the "binding site".

Table 2 and Figure S14 show that the EM of LRRK2_pS910pS935 is significantly higher $(110 \mathrm{mM})$ than that of the other peptides. Additionally, the binding enthalpy for this ditopic peptide was higher than expected on the basis of the sum of the binding enthalpies of the singly phosphorylated peptides (Table 1). Both facts can be explained by analysis of the crystal structure of the complex of this doubly phosphorylated peptide with 14-3-3 (Figure 4A). The "pS910 site" of the bivalent peptide forms an $\alpha$ helix in the binding groove of 14-3-3. By this exceptionally compact packing, more amino acid residues (Y917 and R918) are visible in the electron density than seen in other 14-3-3 crystal structures (for example, the pS768 site of CFTR in Figure 4B), the binding of which clearly provides a contribution to the interaction. These amino acid residues are theoretically part of the "linker" between the two binding sites and were therefore not included in the design of the singly phosphorylated peptide LRRK2_pS910. As a result, the "linker" between the pS910 and pS935 binding sites actually plays an important role in the binding leading to a higher EM and a higher binding enthalpy. Additionally, these data show that the glycine/serine linkers used to bridge large distances between the binding sites in the LRRK2 peptides (pS860pS910, pS910pS1444, and pS935pS1444) all show low EMs, hinting that they have minimal interaction with the 14-3-3 protein.

Analysis of the sequences of all of the peptides (Tables S1 and S2) revealed no correlation between the isoelectric point, charge, or hydrophilicity/hydrophobicity of the peptides, and the enthalpy/entropy values. Peptides containing proline residue(s), however, show both higher binding enthalpies and higher entropy penalties by binding to 14-3-3 than the peptides that do not contain any proline residues. This is especially clear when looking at the singly phosphorylated peptides of both the CFTR and the LRRK2 protein (Figure 5). A possible explanation is that the presence of a proline residue in the peptide enhances the steric fit into the binding groove of 

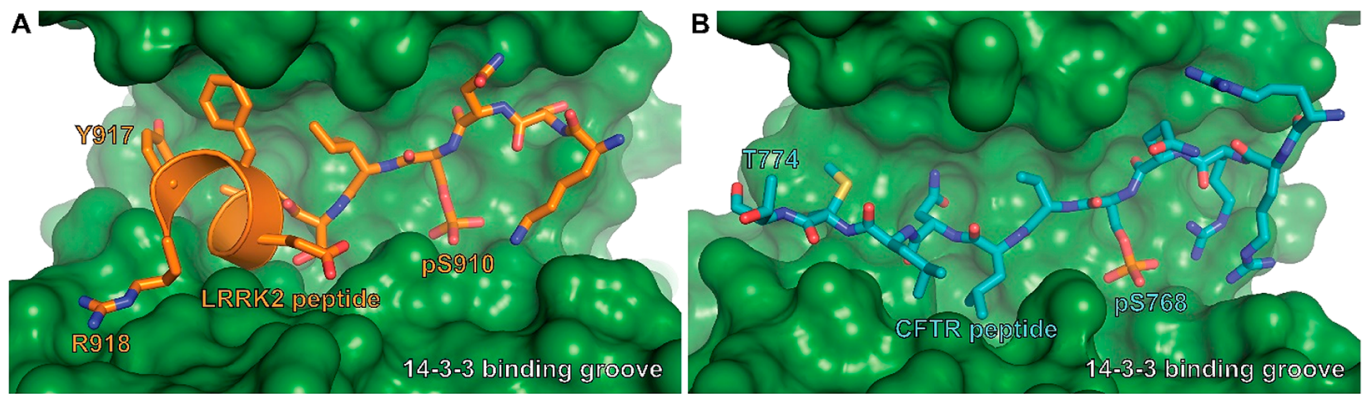

Figure 4. The LRRK2_pS910 site forms an $\alpha$ helix causing more amino acid residues to participate in binding than seen in other 14-3-3 interactions like CFTR_pS768. (A) The pS910 site of the LRRK2_pS910pS935 peptide (orange sticks and cartoon) binding into the binding groove of 14-3-3 $\sigma$ (green surface) (protein data bank (PDB) ID: 5MYC). ${ }^{39}$ (B) The pS768 site of the CFTR_pS753pS768 peptide (cyan sticks) binding into the binding groove of 14-3-3 $\beta$ (green surface) (PDB ID: 6HEP).

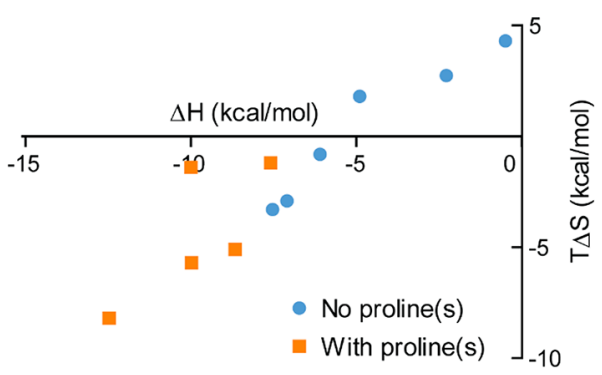

Figure 5. Enthalpy $(\Delta H)$-entropy $(T \Delta S)$ correlation plot of the singly phosphorylated peptides from both LRRK2 and CFTR binding to 14-3-3. The peptides containing proline(s) are colored orange, and peptides not containing proline $(\mathrm{s})$ are colored blue.

14-3-3, causing a higher binding enthalpy via a stronger conformational fixation.

The last observation that stands out is that of all singly phosphorylated peptides of the CFTR protein pS768 is clearly the strongest binder, but has the lowest binding enthalpy for binding to 14-3-3 as well as a positive binding entropy (Table 1 ), which are caused by the $>1$ slope of the enthalpy-entropy compensation plot as explained above. This could be accompanied by secondary structure formation of the peptide in solution, which is lost upon binding to 14-3-3. Also, LRRK2_pS910 and LRRK2_pS973 have positive entropy values. These three peptides have in common that one side of the phosphorylated serine residue is strongly hydrophilic, and the other side hydrophobic, which could cause secondary structure formation in solution. This may imply that some disordered protein systems can contain some degree of secondary structure in the unbound form, the loss of which can result in entropy-enhanced binding of an involved binding site. $^{48}$

Three or More 14-3-3 Binding Sites. Next to the singly and doubly phosphorylated peptides, also a trivalent CFTR peptide containing three neighboring phosphorylated serine residues was synthesized and its binding to 14-3-3 $\beta$ analyzed (CFTR_pS753pS768pS795, Tables S1 and S3). The $K_{\mathrm{d}}$ value of this peptide is slightly lower than that of the strongest binder of the doubly phosphorylated peptides $(7.8 \mu \mathrm{M}$ vs 13 $\mu \mathrm{M}$ of CFTR_pS768pS795). Moreover, the enthalpy and entropy values $(-12.3$ and $-5.2 \mathrm{kcal} / \mathrm{mol})$ nicely fit on the trend line of Figure 3A, confirming ditopic binding with a very similar EM. The enthalpy change of the triply phosphorylated peptide $(-12.3 \mathrm{kcal} / \mathrm{mol})$ does not fit with the sum of any two of the three singly phosphorylated peptides $(-8.67,-2.29$, and $-12.5 \mathrm{kcal} / \mathrm{mol}$, for $\mathrm{pS753}, \mathrm{pS768}$, and pS795, respectively).
When considering that the triply phosphorylated peptide may form ditopic complexes from a combination of pS753 and pS768 bound to $14-3-3(\Delta H=-11.0 \mathrm{kcal} / \mathrm{mol})$, and pS768 and pS795 bound to $14-3-3(\Delta H=-14.8 \mathrm{kcal} / \mathrm{mol})$, it is clear that the observed enthalpy change is between that of these two ditopic peptide motifs.

The possible equilibria for the binding of a trivalent phosphorylated construct to the bivalent 14-3-3 host are described in the model displayed in Figure S4A. pS768 is the logical pivot in the interaction, because (i) the individual interaction of this site is the strongest as compared to pS753 and pS795, and (ii) the site is located in between the other two, and therefore the EM values of the two ditopic interactions involving pS768 can be assumed to be higher than that of the joint binding of pS753 and pS795 (although the discussion above has indicated that the distance dependence is probably limited). The overall affinity can be written as the sum of the ditopic affinities, as shown in Figure S4B. When converting this back to the $K_{\mathrm{d}}$ value, the calculated $K_{\mathrm{d}}$ value $(9.2 \mu \mathrm{M})$, resulting from the summation of the interactions for CFTR_pS753pS768 and CFTR_pS768pS795, is in good agreement with the experimental value $(7.8 \mu \mathrm{M})$. Moreover, the ratio between the contributing ditopic affinities is also the ratio in which the ditopic motifs are expected to occur in the tripeptide, that is, approximately $70 \%$ of pS768pS795 and $30 \%$ of pS753pS768. When taking these values to calculate the weighted average of the ditopic enthalpy contributions, a calculated $\Delta H$ of $-13.7 \mathrm{kcal} / \mathrm{mol}$ is found, which is, as expected, between the values of the ditopic peptides, and is in reasonable agreement with (although slightly more exothermic than) the experimental value for the triply phosphorylated peptide. When one would only take the enthalpy values to estimate the contributions of pS768pS795 and pS753pS768, one arrives at a 35:65 ratio, but the error in the enthalpy values is too large to make a very accurate assessment. When attempting to also incorporate a potential minor contribution of pS753pS795, several assumptions need to be made. A $K_{d}$ value of $0.37 \mathrm{mM}$ for pS753pS795 binding to 14-3-3 is obtained by using the values obtained by FP (Table S3) and scaling it to allow comparison with the other $K_{\mathrm{d}}$ values obtained by ITC. A strongly exothermic enthalpy of -21.2 $\mathrm{kcal} / \mathrm{mol}$ can be estimated from the values interpolated from the ditopic peptides. Even when assuming an EM (20 mM) similar to that of the other ditopic peptide motifs, the contribution of this ditopic motif to the overall binding is less than $5 \%$. Moreover, the calculated binding enthalpy for the tripeptide becomes even more exothermic $(-14.1 \mathrm{kcal} / \mathrm{mol})$, 


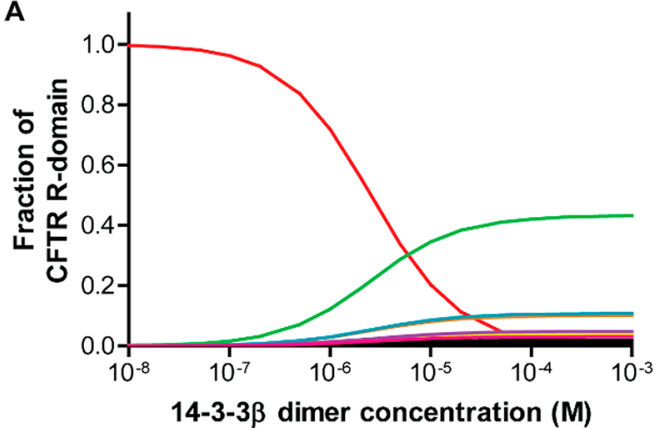

- Not bound pS712+pS768

- pS753+pS768

- pS737+ pS768

- pS768+pS795

- pS768+pS813

- pS795 + pS813
B

\begin{tabular}{c|c} 
Binding sites & Fraction \\
\hline$p S 768+p S 795$ & 0.432 \\
\hline$p S 737+p S 768$ & 0.108 \\
\hline$p S 753+p S 768$ & 0.102 \\
\hline$p S 768+p S 813$ & 0.048 \\
\hline$p S 712+p S 768$ & 0.038 \\
\hline$p S 795+p S 813$ & 0.032
\end{tabular}

Figure 6. Simulation of the species distribution of the fully phosphorylated CFTR R-domain binding to 14-3-3 $\beta$. The simulation takes only the binding of one 14-3-3 dimer into account, uses the measured EM values for all measured ditopic peptides, and assumes a weak $\left(r^{-3 / 2}\right)$ distance dependence for the other EM values. (A) The fraction of R-domain bound to 14-3-3 $\beta$ at different concentrations of the 14-3-3 $\beta$ dimer. The combinations which represent $>3 \%$ of the total R-domain are colored, and the rest is displayed in black. (B) The fractions of the different complexes, at a 14-3-3 dimer concentration of $1 \mathrm{mM}$ (all fractions $>3 \%$ are shown).

while without assuming any contribution from this motif it is already more exothermic than experimentally observed. Therefore, it can be concluded that the ditopic motif pS753pS795 is not contributing significantly to the binding of the tritopic CFTR_pS753pS768pS795, in agreement with the initial assumption described above. Overall, it can concluded that both the affinity values and the binding enthalpies indicate the presence of sizable contributions of both ditopic motifs pS753pS768 and pS768pS795 to the binding of the tripeptide, but none of the motif (pS753pS795) that does not involve pS768. Overall, both the enthalpies and the free energies indicate involvement of both neighboring ditopic motifs in the binding around a pivot (here, pS768). This behavior may be a general rule in multitopic disordered proteins to boost affinity and to increase binding selectivity for a particular site.

The fully phosphorylated CFTR R-domain, featuring up to nine phosphorylated binding sites, was obtained via coexpression with PKA kinase. The interaction between this protein domain and $14-3-3 \beta$ was measured with both microscale thermophoresis (MST) and ITC (see Table S3). The apparent $K_{\mathrm{d}}$ value estimated from ITC $(42 \mu \mathrm{M})$ is slightly weaker than that of the strongest dimers. While one would maybe at first glance expect the full R-domain to bind stronger, the value is most likely within experimental error, identical to what can be expected from a summation of all possible ditopic motifs. Furthermore, a not fully complete degree of phosphorylation as visible in Figure S15, which would limit certain ditopic motifs in fractions of the R-domain molecular population, should be taken into account. The $K_{d}$ value determined by MST $(3.8 \mu \mathrm{M})$ is lower, and agrees fairly well with the values obtained by FP for the di- and triphosphorylated constructs. The binding enthalpy of the full R-domain to $14-3-3 \beta$ is more exothermic $(-16.4 \mathrm{kcal} / \mathrm{mol})$ than that of the strongest binding doubly phosphorylated peptide CFTR_pS768pS795 $(-14.8 \mathrm{kcal} / \mathrm{mol})$ or the triply phosphorylated peptide CFTR_pS753pS768pS795 (-12.3 kcal/ $\mathrm{mol}$ ). There is one ditopic motif (CFTR_pS795pS813) for which an even more exothermic value was observed $(-20.0$ $\mathrm{kcal} / \mathrm{mol}$ ), so the strongly exothermic value for the R-domain may imply a contribution of this motif. Yet, the list of enthalpy values of possible ditopic motifs (Table S3) is incomplete, so contributions of other motifs can at this stage not be excluded. On the basis of the dimeric $K_{d}$ values, pS795pS813 is not expected to contribute more than $6 \%$, while a contribution of more than $40 \%$ would be needed to explain the more exothermic enthalpy observed for the R-domain. Therefore, most likely, the strong dimeric binders pS753pS768 and pS768pS795 still play an important role in the binding of the R-domain, but minor contributions of other ditopic motifs are probably present as well.

Binding Simulations of the Entire Protein Domains. The $K_{\mathrm{d}}$ values and EMs of the different binding sites in the proteins can be used to simulate the binding modes (i.e., which site interacts to what fraction, and in which species) of the whole R-domain of CFTR or the LRRK2 protein to one 14-3-3 dimer. To do so, it is assumed that all binding sites in the proteins are phosphorylated, and every combination of binding sites is able to bind to 14-3-3. Additionally, the assumption is made that the 14-3-3 concentration is much higher than the concentration of CFTR or LRRK2. It is well-known that 14-33 proteins are very abundant in the human cell (up to $1-2 \%$ of the total amount of soluble protein, dependent on cell type), ${ }^{49}$ and are thus most probably present in higher much concentrations than CFTR and LRRK2. In this simulation, there are 45 different ( 9 monovalent and 36 divalent) modes for the phosphorylated CFTR R-domain to bind to one 14-3-3 dimer. The $K_{\mathrm{d}}$ values of all of the phosphorylated binding sites have been measured (Table S3), and the EMs of the neighboring binding sites were calculated on the basis of the $K_{\mathrm{d}}$ values of the doubly phosphorylated peptides and singly phosphorylated peptides (Table 2). Because the EM values of the non-neighboring binding sites are unknown, different scenarios for the complete set of EM values are considered.

In the first scenario, the values of the EMs of the divalent motifs are taken as measured, and the assumption is made that the remaining EMs are weakly $\left(r^{-3 / 2}\right)$ distance dependent, taking $\mathrm{EM}=10 \mathrm{mM}$ when 20 connecting amino acid residues are present between the phosphorylated serine residues, and $\mathrm{EM}=10 \times\left(N_{\mathrm{aa}} / 20\right)^{-3 / 2}($ in $\mathrm{mM})$, where $N_{\mathrm{aa}}$ is the number of amino acid residues between the phosphorylated serine residues. The resulting distribution between the 45 different possible binding complexes in this situation is displayed in Figure 6A. The results show that the R-domain will start binding to 14-3-3 at a 14-3-3 dimer concentration of around $100 \mathrm{nM}$, and most of the R-domain will be bound to 14-3-3 at a concentration higher than $2.5 \mu \mathrm{M}$, which is close to the $K_{\mathrm{d}}$ value of the strongest doubly phosphorylated binder $(2.9 \mu \mathrm{M})$. Even though 45 possible complexes can be formed in the simulation, some species clearly stand out as major 
A

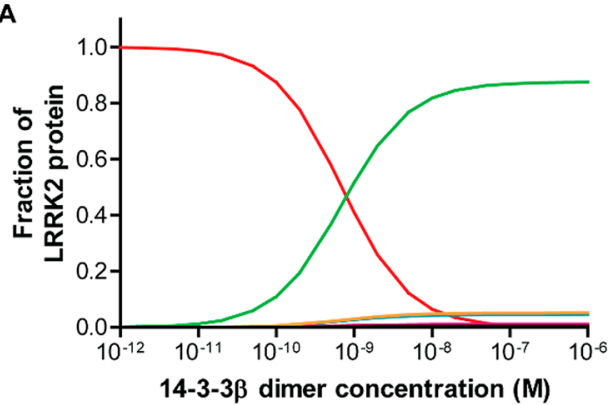

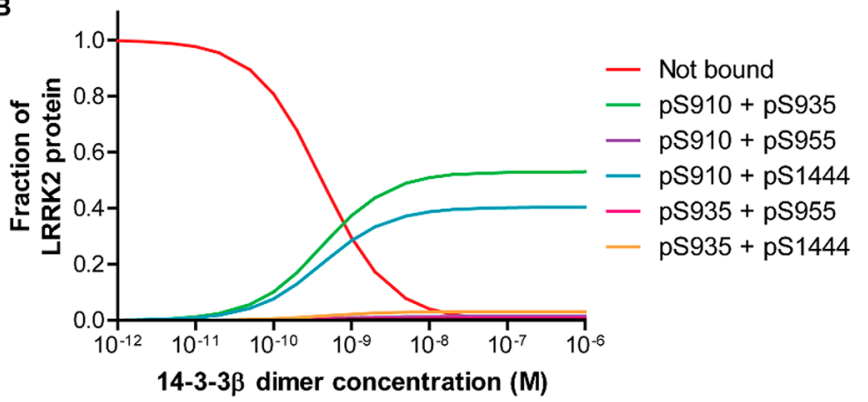

c

\begin{tabular}{c|c|c} 
Binding sites & Fraction (sc. A) & Fraction (sc. B) \\
\hline pS910 + pS935 & 0.875 & 0.530 \\
\hline pS935 + pS1444 & 0.052 & 0.031 \\
\hline pS910 + pS1444 & 0.046 & 0.403 \\
\hline$p S 935+p S 955$ & 0.011 & 0.007 \\
\hline$p S 910+p S 955$ & 0.006 & 0.014
\end{tabular}

Figure 7. Simulations of the species distribution of the fully phosphorylated LRRK2 protein binding to 14-3-3 $\gamma$. The simulations take only the binding of one 14-3-3 dimer into account, use the measured EM values for all measured ditopic peptides, and assume a weak ( $r^{-3 / 2}$ ) distance dependence for the other EM values. (A) The fraction of LRRK2 bound to 14-3-3 $\gamma$ at different concentrations of the 14-3-3 $\gamma$ dimer in the scenario of the measured $K_{d}$ value of LRRK2_pS910 and EM of pS910/pS935. (B) The fraction of LRRK2 bound to 14-3-3 $\gamma$ at different concentrations of the 14-3-3 $\gamma$ dimer in the scenario of the $K_{\mathrm{d}}$ value of LRRK2_pS910 from the literature, ${ }^{50}$ resulting in a lower EM for pS910/pS935. The combinations that represent $>1 \%$ of the total LRRK2 protein are colored, and the others are displayed in black. ( $\mathrm{C}$ ) The fractions of the different complexes, at a 14-3-3 dimer concentration of $1 \mathrm{mM}$, of the scenarios shown in (A) and (B) (all fractions $>1 \%$ are shown).

contributors (Figure 6). The major fraction of the R-domain (43.2\%) binds to $14-3-3$ by the combination of the pS768 and pS795 binding sites (Figure 6B). Subsequently, the p5737/ pS768 and the pS753/pS768 combinations represent $10.8 \%$ and $10.2 \%$ of the R-domain binding to $14-3-3$, respectively. Other minor contributors are the pS768/pS813 and pS712/ pS768 combinations ( $4.8 \%$ and $3.8 \%$ ), and all others are $<3 \%$. As expected, a total sum of $77.3 \%$ of the R-domain binds, in this scenario, in a way that pS768 is involved. The largest fraction in which pS768 is not involved is the pS795/pS813 combination, representing only $3.2 \%$ of complex formed.

Alternatively, when the EM values of all non-neighboring binding sites are kept at a constant value of $5 \mathrm{mM}$ (equal to $\mathrm{EM}_{\mathrm{ov}}$ ), as inspired by the observed absence of a clear relationship between the EM values as discussed above, the binding between the CFTR R-domain and the 14-3-3 dimer is still dominated by the pS768/pS795 combination, albeit to a somewhat lesser extent $(29.6 \%)$. As a result, more species are contributing to the overall speciation. The next most abundant combinations are then pS737/pS768, pS753/pS768, pS670/ pS768, pS712/pS768, and pS768/pS813 (with 7.1\%, 7.0\%, $6.7 \%, 6.1 \%$, and $5.6 \%$, respectively). Also, in this scenario, the pS768 site is the most important binder, and pS768/pS795 remains the most strongly expressed divalent motif.

In a third scenario, only neighboring sites are taken into account; that is, the EM values of all other combinations are set to zero. In this case, the binding is dominated, even more than in the scenario shown in Figure 6, by the pS768/pS795 combination $(65.1 \%)$. The only other species in this scenario that score $>3 \%$ are pS753/pS768 (15.3\%) and pS795/pS813 $(4.8 \%)$, whereas over $82 \%$ of the species contain pS768. Overall, therefore, it can be concluded that, regardless of the scenario for the EM values, the binding of the R-domain is strongly dominated by pS768, and primarily by the pS768/ pS795 ditopic motif.

For the hexa-phosphorylated LRRK2 protein, there are fewer ways possible to bind to 14-3-3 (6 monotopic and 15 ditopic ways). That the more distant location of the pS1444 site is located in a totally different protein domain makes for an intriguing case to more closely evaluate the effect of the EM value for combinations including this binding site. When the EMs of the LRRK2 protein are assigned in a weakly $\left(r^{-3 / 2}\right)$ distance-dependent manner, the simulation results in binding combinations as depicted in Figure 7A. In this scenario, the binding of the LRRK2 protein to 14-3-3 starts at a significantly lower concentration $(10 \mathrm{pM})$ than that of the CFTR protein, and most of the LRRK2 protein will be bound to 14-3-3 at a concentration of $0.69 \mathrm{nM}$, which is close to the $K_{\mathrm{d}}$ value of the strongest doubly phosphorylated binder $(0.28 \mathrm{nM})$. The explicit major fraction of the LRRK2 protein $(78.5 \%)$ binds with the combination of pS910 and pS935 to 14-3-3 (Figure 7, scenario A). Additionally, minor fractions of $\mathrm{pS} 935 / \mathrm{pS} 1444$ and $\mathrm{pS} 910 / \mathrm{pS} 1444$ are binding to $14-3-3$ (5.2\% and $4.6 \%$, respectively).

Figure $7 \mathrm{~B}$ visualizes a similar scenario, with a correction for the high EM value of LRRK2_pS910pS935 based on peptide binding data published by Muda et al. $\left(K_{\mathrm{d}, \mathrm{pS} 910}=661 \mathrm{nM}\right.$ and $\mathrm{EM}_{\mathrm{pS910pS935}}=7.6 \mathrm{mM}$; see the Supporting Information for details). ${ }^{50}$ This scenario shows again that the major fraction of the LRRK2 protein (53.0\%) binds with the combination of pS910 and pS935 to 14-3-3; however, the fraction of the pS910/pS1444 combination increased significantly to $40.3 \%$, while the pS935/pS1444 combination decreased slightly to $3.1 \%$.

In contrast to the CFTR case, setting the EM of all of the non-neighboring binding sites in LRRK2 to the $\mathrm{EM}_{\mathrm{ov}}$ of LRRK2 of $10 \mathrm{mM}$, the simulation outcome is completely different. The major fraction of the LRRK2 protein is then bound by the pS935/pS1444 and pS910/pS1444 binding site combinations to $14-3-3$ ( $45.7 \%$ and $43.2 \%$, respectively), and only a minor fraction by pS910/pS935 (6.0\%). When the alternative parameters $K_{\mathrm{d}, \mathrm{pS} 910}$ and $\mathrm{EM}_{\mathrm{pS} 910 \mathrm{pS} 935}$ are used for the calculations, the major fraction is pS910/pS1444 (91.6\%),

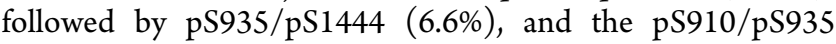




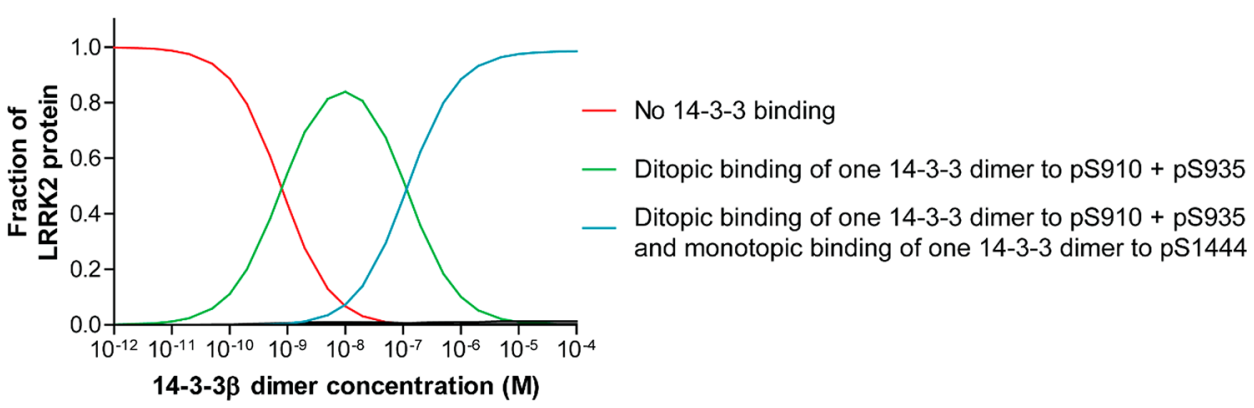

Figure 8. Simulation of the species distribution of the fully phosphorylated LRRK2 protein binding to two 14-3-3 $\gamma$ dimers. The simulation takes the binding of one and two 14-3-3 dimers into account, uses the measured EM values for all measured ditopic peptides, and assumes all EM values for 1444 to be 0 . The graph shows the fractions of LRRK2 binding to one or two 14-3-3 $\gamma$ dimers at different concentrations of the 14-3-3 $\gamma$ dimer in the scenario of the measured $K_{\mathrm{d}}$ value of LRRK2_pS910.
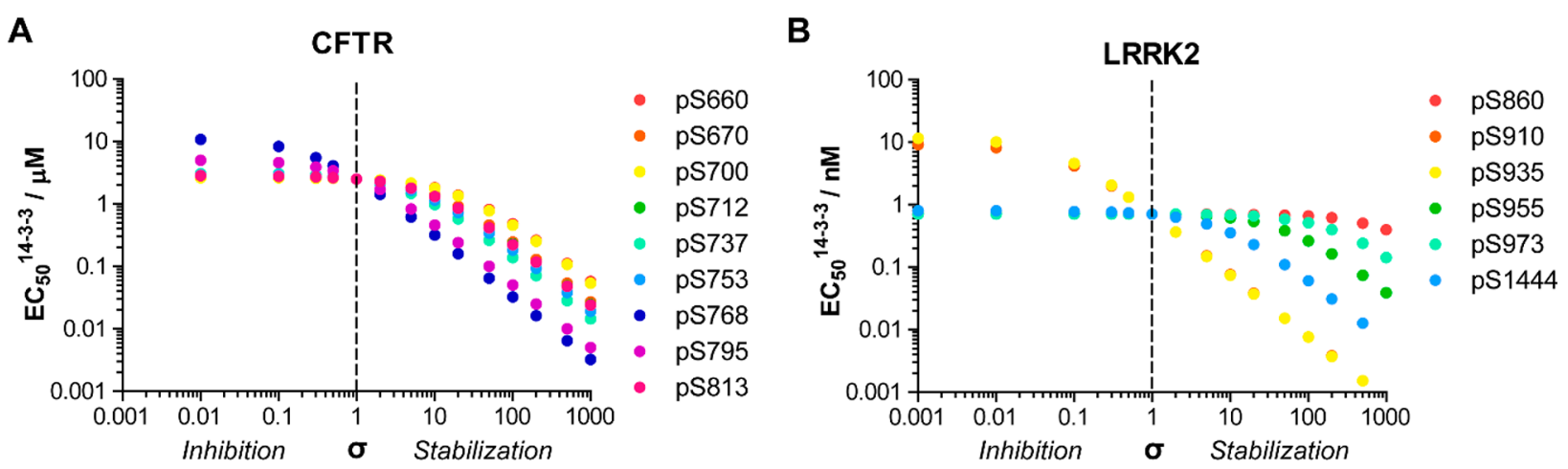

Figure 9. Simulated effect of modulation of a single binding site. $\mathrm{EC}_{50}$ of simulated titrations 14-3-3 to CFTR and LRRK2 upon modulation of single binding sites by a factor $\sigma$. When $\sigma=1$, the affinity is unchanged, dashed line.

combination represents only $0.9 \%$. Even though an EM of 10 $\mathrm{mM}$ for the pS1444-based ditopic motifs is probably too high, the example shows the strong influence of only one of many phosphorylation sites on the overall binding event and stimulates considering the high impact of the protein folding characteristics on distances, EMs, and binding affinities of such multivalent PPIs.

In the opposite situation that $\mathrm{pS} 1444$ is physically not able to bind to 14-3-3 in combination with any of the other binding sites due to conformational restrictions, both scenarios have major fractions of the $\mathrm{pS} 910 / \mathrm{pS} 935$ combination present (97.6\% and $94.2 \%$ ), confirming that pS1444 is the only site that has a significant influence apart from the pS910/pS935 motif. Interestingly, when the simulation in this case is expanded toward the possibility of two 14-3-3 dimers binding to one LRRK2 protein, the fraction $\mathrm{pS910/pS935} \mathrm{is} \mathrm{still}$ dominant, but when the concentration 14-3-3 increases above a concentration of $1 \mathrm{nM}$ a second 14-3-3 protein starts binding (monotopically) at the pS1444 site (Figure 8). Subsequently,

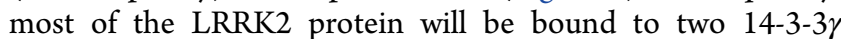
dimers at a 14-3-3 dimer concentration of $125 \mathrm{nM}$, which is close to the $K_{\mathrm{d}}$ value of the singly phosphorylated pS1444 peptide $(110 \mathrm{nM})$.

Implications of the Multivalent Model for Protein Point Mutations and Molecular Modulation. The thermodynamic models and resulting simulations can be used to provide information on the importance of individual binding sites in multivalent PPIs, as shown above. Similarly, the multivalent model can be used to predict the effects on the PPI when individual binding sites are being modulated. Both scenarios in which the binding site is being weakened or removed, for example, via a small-molecule inhibitor, a point mutation, or an enzymatic dephosphorylation, can be considered as well as scenarios in which individual binding sites are strengthened, for example, via a small-molecule stabilizer or a stabilizing mutation in a flanking amino acid.

For the case of binding site removal, it is interesting to consider the CFTR pS768 acting as a pivot in the PPI interaction. When the pS768 is removed from the CFTR simulation as described in Figure 6, the overall 14-3-3 binding is taken over by many different combinations of binding sites, the most dominant being pS795/pS813 (14.0\%), pS670/ pS700 (8.5\%), pS670/pS712 (8.4\%), pS660/pS700 (5.5\%), and $\mathrm{pS} 737 \mathrm{pS} 795$ (5.3\%). Where the $\mathrm{pS} 795 / \mathrm{pS} 813$ combination is already the most prominent binding combination without pS768, in the regular case, in the absence of pS768 it becomes the most prominent interaction overall. The absence of a very prominent pivot interaction leads to a broader set of interaction bis phosphorylated motives to the overall complex formation. Interestingly, the deletion of the pS768 interaction leads only to a decrease in binding affinity of the full R-domain for $14-3-3 \beta$ from 2.5 to $11 \mu \mathrm{M}$. The single removal of any of the other binding sites leads to an even smaller decrease in binding affinity between $2.7 \mu \mathrm{M}$ (for pS660) and 5.1 $\mu \mathrm{M}$ (for pS795). The simulations thus make clear that a point mutation or dephosphorylation of one of the binding sites does not cause a large effect on the ability of the multivalent protein to bind to the bivalent 14-3-3 platform. The deletion of one binding site is thus for a major part rescued by combinations of the other binding sites. These observations also argue for a role of the multiphosphorylation of the $\mathrm{R}$ domain in generating ultrasensitive responses in binding to the 14-3-3 protein. 

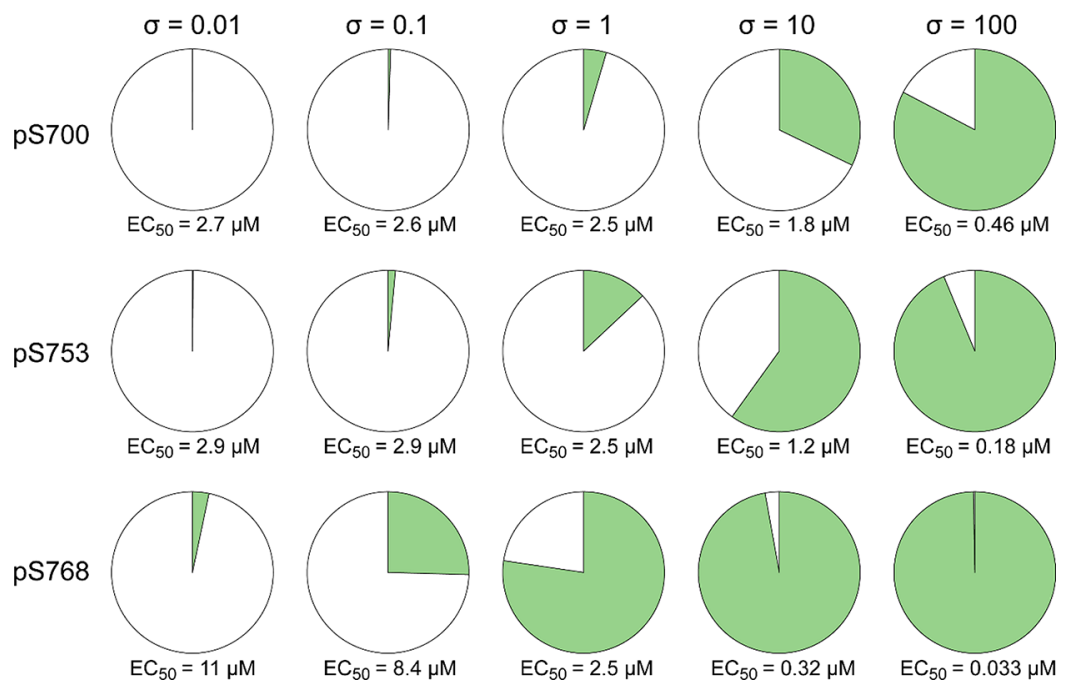

Figure 10. Simulation of the species distribution of the fully phosphorylated CFTR R-domain binding to 14-3-3 $\beta$ at different $\sigma$ values (0.01, $0.1,1$, 10, and 100) for pS700, pS753, and pS768. The pie charts show the fraction of the R-domain that is bound to 14-3-3 including the particular binding site (green) versus the fraction that binds not using this site (white). The $\mathrm{EC}_{50}$ values are representing the binding of the $\mathrm{R}$-domain to 143-3.

We also simulated the effect of small-molecule PPI modulation of each individual binding site on the overall affinity between the two proteins (Figures 9 and 10). We systematically changed the $K_{\mathrm{d}}$ of each individual binding with a factor $\sigma$, ranging between 0.001 and 1000 . When $\sigma$ is $<1$, the interaction is inhibited; when $\sigma$ is $>1$, the affinity is increased. Figure 9 depicts progression of the global half maximal effective concentration $\left(\mathrm{EC}_{50}\right)$ of 14-3-3 to either CFTR or LRRK2 upon a local change in affinity for each binding site. For CFTR, a decrease in affinity of pS768 and pS795 causes a drop in global affinity, in line with the results and values described above. The weaker effect of inhibition of the other sites on the overall $\mathrm{EC}_{50}$ also becomes clear from these figures. For LRRK2, the effects of inhibition of either pS910 or pS935 are most prominent, while changes of other sites have almost no effect on the overall $\mathrm{EC}_{50}$. The inhibition of either of these two sites leads to a more pronounced weakening in PPI formation as compared to individual site mutations in CFTR. These observations are in line with the experiments described in Figure 7A, which revealed the pS910 or pS935 combination alone to contribute to $88 \%$ of the total complex with 14-3-3. In contrast to CFTR, which features one pivot in the complex formation, for LRRK2 two sites are equally important.

For small-molecule stabilization of such multivalent PPIs, it is equally important to delineate the effects of individual site stabilization on the overall affinity of the PPI. The simulations for both CFTR and LRRK2 show that the increase of the affinity of the dominating sites has the most potent effect on the overall PPI stabilization (Figure 9). Stabilization of the other, weaker binding, sites can also affect the affinity of the PPI complex, to a much stronger degree than their inhibition. Nevertheless, for these weaker binding sites, a larger $\sigma$ is required to result in a noticeable stabilization of the complex: these sites first have to become dominating before there is a global effect on the $\mathrm{EC}_{50}$. Drug discovery endeavors aiming for the discovery of small-molecule stabilizers of such multivalent PPIs would thus probably best consider targeting one of the already strongly binding interaction sites, as small changes in their affinities quickly lead to relevant changes in the $\mathrm{EC}_{50}$ of the PPI. This is especially prominent for the LRRK2 case featuring the two key interaction motives pS910 and pS935. For CFTR the differences between the individual sites are less pronounced, potentially providing also room for the stabilization of other interaction sites beyond pS768.

The conceptual differences in term of importance and overall effect between the inhibition and stabilization of binding sites are illustrated for three different binding site of CFTR in Figure 10. Here, the contributions of each site to the overall $\mathrm{EC}_{50}$ of the PPI and the fraction of that site bound to 14-3-3 are shown. The results clearly show that inhibition of each site has overall very little effect on the $\mathrm{EC}_{50}$ of PPI. Even for the case of pS768, where the fraction of this site contributing to complex formation strongly diminishes upon lowering the $\sigma 100$-fold, the $\mathrm{EC}_{50}$ only lowers less than 5-fold. Stabilization of individual sites, on the other hand, results in much more pronounced effects on the overall $\mathrm{EC}_{50}$. Already the 100-fold strengthening of the pS700 site, not strongly contributing under normal conditions, leads to this site being the major species involved in complex formation and a 5-fold enhancement of the $\mathrm{EC}_{50}$. A similar stabilization of the pivot pS768 site enhances the $\mathrm{EC}_{50}$ even 75 -fold. These results thus bring forward that for multivalent PPIs the stabilization of individual interaction sites is significantly more effective than inhibition, bringing forward clear potential for the development of small-molecule stabilizers in PPI drug discovery.

\section{CONCLUSIONS}

In this Article, we have brought forward a thermodynamic model, based on a ditopic host-guest system, describing multivalent PPIs and featuring the EM as the prime model parameter governing the intramolecular binding step in the divalent interactions. Fitting of binding data of two exemplary model systems, the interaction of the bivalent 14-3-3 protein scaffold with both the nonavalent CFTR and the hexavalent LRRK2 protein, with the model allowed one to determine the underlying thermodynamics, revealing a strong enthalpyentropy correlation in both systems. The thermodynamic model also allows one to explore the effect of different scenarios of EM distributions. This is especially relevant when considering, for example, that the individual binding sites of 
LRRK2 are located within two ordered domains, and that the disordered domain of CFTR, on which the phosphorylated binding sites are located, is located close to the plasma membrane and connected to two more rigid particles. All of these factors will put restrictions on the possible conformations of the domains, and therefore the possible combinations of sites binding to 14-3-3 and overall affinity of the complexes and their regulation.

The binding enthalpy-entropy correlation of the CFTR peptides binding to $14-3-3 \beta$ showed that a change in binding enthalpy is compensated by a change in entropy, which resulted in very similar $K_{d}$ values of the different peptides. Even though for the CFTR interaction with 14-3-3, the pS768 acts as a pivot, simulations of the binding of the full CFTR Rdomain revealed that the removal of this or any other single binding site leads to only small effects on the overall binding affinity. In the cell, this thermodynamic principle could act as a mechanism protecting the CFTR protein from losing its ability to bind to 14-3-3 due to point mutations or dephosphorylation of a certain binding site.

The binding enthalpy-entropy compensation effect for LRRK2 is much smaller, as compared to CFTR, and concomitantly there is a larger difference in $K_{d}$ values of the individual binding sites for 14-3-3. As a result, the two binding sites pS910 and pS935 dominate the complex formation of LRRK2 with 14-3-3. Still, the distantly located pS1444 site, depending on the EM, could also play a crucial role in the complex formation. Depending on the folding of the full protein, to be resolved with structural studies, the pS1444 site could be in close proximity to the other sites and significantly contribute to the PPI formation.

The thermodynamic model was finally used to analyze and predict the effects of the modulation of individual sites on the strength of the multivalent PPIs. The results clearly bring forward that for these multivalent PPIs there is a conceptual difference between the inhibition and stabilization of individual binding sites, in terms of both importance and resulting overall effect. The stabilization of the most prominent individual interaction sites is significantly more effective in eliciting a relevant change in the overall binding affinity of the PPI, than the inhibition of a single interaction site.

The conceptual thermodynamic binding model described provides a long desired general framework for describing multivalent PPIs. The model allows for in-depth understanding of the underlying parameters of such complex PPIs and simultaneously has strong value for guiding the development of small-molecule modulators in PPI drug discovery. Particularly the small-molecule stabilization of PPIs can be strongly steered via this model as an entry for drug discovery for multivalent PPIs. We envision that, with appropriate adjustments, the thermodynamic analysis and resulting implications as described here can be translated to many other multivalent PPI systems and help in achieving their highly needed fundamental understanding and to address the underlying molecular challenges in pathologies and drug development.

\section{EXPERIMENTAL SECTION}

Peptide Synthesis. The phosphorylated peptides were synthesized via Fmoc solid-phase peptide synthesis making use of an Intavis MultiPep RSi peptide synthesizer. ${ }^{51-53}$ The singly phosphorylated peptides were synthesized on Rink amide resin (Novabiochem; 0.59 $\mathrm{mmol} / \mathrm{g}$ loading) and the doubly and triply phosphorylated peptides on TentaGel R RAM resin (Rapp Polymere; $0.18 \mathrm{mmol} / \mathrm{g}$ ). To increase the peptide synthesis yield of the longer doubly and triply phosphorylated peptides, pseudoproline dipeptide residues (FmocGln(Trt)-Ser(psiMe,Mepro)-OH, Fmoc-Ile-Ser(psiMe,Mepro)-OH, Fmoc-Asp(OtBu)-Ser(psiMe,Mepro)-OH, Fmoc-Leu-Ser(psiMe,Mepro)-OH, and Fmoc-Ala-Ser(psiMe,Mepro)-OH, Novabiochem) were incorporated. The peptides used for ITC and crystallization were $\mathrm{N}$-terminally acetylated before deprotection and cleavage of the resin. The peptides used in the FP assays were labeled via an Fmoc-O1Pen-OH linker (Iris Biotech $\mathrm{GmbH}$ ) with FITC (Sigma-Aldrich).

The peptides were purified using a preparative LC-MS system that was comprised of a LCQ Deca XP Max (Thermo Finnigan) ion-trap mass spectrometer equipped with a Surveyor autosampler and Surveyor photodiode detector array (PDA) detector (Thermo Finnigan). Solvents were pumped using a high-pressure gradient system using two LC-8A pumps (Shimadzu) for the preparative system and two LC-20AD pumps (Shimadzu) for the analytical system. The crude mixture was purified on a reverse-phase $\mathrm{C} 18$ column (Atlantis T3 prep OBD, $5 \mu \mathrm{m}, 150 \times 19 \mathrm{~mm}$, Waters) using a flow of $20 \mathrm{~mL} \mathrm{~min}^{-1}$ and linear acetonitrile gradient in water with $0.1 \% \mathrm{v} / \mathrm{v}$ trifluoracetic acid (TFA). Fractions with the correct mass were collected using a PrepFC fraction collector (Gilson Inc.). The purity and exact mass of the synthesized peptides were determined using analytical LC-MS. The mass off all peptides corresponded with the calculated mass, with a purity $>90 \%$.

Expression of 14-3-3. His $_{6}$-tagged 14-3-3 proteins were expressed in NiCo21(DE3) competent cells with a pPROEX $\mathrm{HTb}$ plasmid $\left(0.4 \mathrm{mM}\right.$ IPTG, overnight at $\left.18{ }^{\circ} \mathrm{C}\right)$, and purified using $\mathrm{Ni}^{2+}$. affinity chromatography. The proteins were dialyzed against FP or ITC buffer before usage (recipes described below). The 14-3-3 proteins for MST were treated with TEV-protease to cleave off the $\mathrm{His}_{6}$-tag, followed by a second $\mathrm{Ni}^{2+}$-affinity column and size exclusion chromatography.

Fluorescence Polarization Assay. The FITC-labeled peptides were dissolved in FP buffer (10 mM HEPES pH7.4, $150 \mathrm{mM} \mathrm{NaCl}$, $0.1 \%$ Tween $20,1 \mathrm{mg} / \mathrm{mL} \mathrm{BSA)}$ to a final concentration of $100 \mathrm{nM}$ (CFTR peptides and singly phosphorylated LRRK2 peptides), $5 \mathrm{nM}$ (LRRK2_pS860pS910, LRRK2_pS935pS955, and LRRK2 pS955pS973), or $0.5 \mathrm{nM}$ (LRRK2 pS910pS935, LRRK2_pS910pS1444, and LRRK2_pS935pS144). In these solutions, a 2-fold dilution series of 14-3-3 were made in Corning Black round bottom 384-well plates or Grainer Black flat bottom 96-well plates (for LRRK2_pS910pS935, LRRK2_pS910pS1444, and LRRK2_pS935pS144), and their polarization was measured with a Tecan Infinite F500 plate reader $($ ex. $=485 \mathrm{~nm}$, em. $=535 \mathrm{~nm})$. Shown in Figures S6, S7, S9, and S10 are the mean of three experiments.

Isothermal Titration Calorimetry. The ITC measurements were performed with the Malvern MicroCal $\mathrm{iTC}_{200}$. The protein and peptides were dissolved in ITC-buffer ( $25 \mathrm{mM}$ HEPES $\mathrm{pH}$ 7.4, 100 $\mathrm{mM} \mathrm{NaCl}, 10 \mathrm{mM} \mathrm{MgCl} 2,0.5 \mathrm{mM}$ TCEP). One or two times 18 titrations of $2 \mu \mathrm{L}$ were performed at $37^{\circ} \mathrm{C}$ (reference power, $5 \mu \mathrm{Cal} /$ $\mathrm{s}$; initial delay, $60 \mathrm{~s}$; stirring speed, $750 \mathrm{rpm}$; spacing, $180 \mathrm{~s}$ ). In case of two titration series, the data were merged with ConCat32 software. Data are shown in Figures S8, S11, and S12.

Expression of CFTR R-Domain. $\mathrm{N}$-terminally $\mathrm{His}_{6}-\mathrm{SUMO}$ tagged CFTR R-domain was coexpressed with SUMO-tagged PKA from pCDF-duet plasmids in NiCo21(DE3) competent cells (0.2 mM IPTG, overnight at $18{ }^{\circ} \mathrm{C}$ ), and purified using $\mathrm{Ni}^{2+}$-affinity chromatography. For ITC, the $\mathrm{His}_{6}{ }_{6} \mathrm{SUMO}$ tag was removed using dtUD1 protease and a second $\mathrm{Ni}^{2+}$-affinity chromatography purification step. The proteins were dialyzed against ITC buffer before use.

Qtof-MS Analysis. Purity and exact mass of the CFTR R-domain were determined using a high-resolution LC-MS system consisting of a Waters ACQUITY UPLC I-Class system coupled to a Xevo G2 Quadrupole Time of Flight (Q-ToF). The system was comprised of a Binary Solvent Manager and a Sample Manager with Fixed-Loop (SM-FL). The protein was separated $\left(0.3 \mathrm{~mL} \mathrm{~min}^{-1}\right)$ on a column (Polaris C18A reverse phase column $2.0 \times 100 \mathrm{~mm}$, Agilent) using a 
$15-75 \%$ acetonitrile gradient in water supplemented with $0.1 \% \mathrm{v} / \mathrm{v}$ formic acid before analysis in positive mode in the mass spectrometer. Deconvolution of the $\mathrm{m} / z$ spectra was done using the MaxENT1 algorithm in the Masslynx v4.1 (SCN862) software.

Microscale Thermophoresis. The $\mathrm{His}_{6}$-SUMO-tagged CFTR Rdomain was labeled with the Monolith NT His-Tag RED-tris-NTA labeling Kit, following the included protocol. A two-times dilution series of 14-3-3 $\beta$ was made in the presence of $50 \mathrm{nM}$ labeled CFTR R-domain, which was measured in standard treated capillaries (MOZ002) with the Monolith NT.115 of NanoTemper (LED power, 40\%; MST power, $40 \%$; red LED, rt). The temperature jump was used for analysis.

\section{ASSOCIATED CONTENT}

\section{S Supporting Information}

The Supporting Information is available free of charge on the ACS Publications website at DOI: 10.1021/jacs.8b09618.

Extensive clarification of the thermodynamic model; exact peptide and protein sequences used and analytical data; equations and data fitting; tabular listing of thermodynamic parameters; protein crystallography materials and methods; extensive clarification on effective molarity and linker length; and binding simulations (PDF)

\section{AUTHOR INFORMATION}

\section{Corresponding Authors}

*j.huskens@utwente.nl

*l.brunsveld@tue.nl

\section{ORCID $\odot$}

Christian Ottmann: 0000-0001-7315-0315

Jurriaan Huskens: 0000-0002-4596-9179

Luc Brunsveld: 0000-0001-5675-511X

\section{Notes}

The authors declare no competing financial interest.

\section{ACKNOWLEDGMENTS}

This work was funded by The Netherlands Organization for Scientific Research (NWO) via the Gravity Program 024.001.035, Graduate Program 2012 022.004.027, and VICI grant 016.150 .366 .

\section{REFERENCES}

(1) Mammen, M.; Choi, S.-K.; Whitesides, G. M. Polyvalent Interactions in Biological Systems: Implications for Design and Use of Multivalent Ligands and Inhibitors. Angew. Chem., Int. Ed. 1998, 37 (20), 2754-2794.

(2) Fasting, C.; Schalley, C. A.; Weber, M.; Seitz, O.; Hecht, S.; Koksch, B.; Dernedde, J.; Graf, C.; Knapp, E.-W.; Haag, R. Multivalency as a Chemical Organization and Action Principle. Angew. Chem., Int. Ed. 2012, 51 (42), 10472-10498.

(3) Ferrell, J. E., Jr; Ha, S. H. Ultrasensitivity Part II: Multisite Phosphorylation, Stoichiometric Inhibitors, and Positive Feedback. Trends Biochem. Sci. 2014, 39 (11), 556-569.

(4) Menche, J.; Sharma, A.; Kitsak, M.; Ghiassian, S. D.; Vidal, M.; Loscalzo, J.; Barabasi, A.-L. Uncovering Disease-Disease Relationships through the Incomplete Interactome. Science 2015, 347 (6224), 1257601-1257601.

(5) Huttlin, E. L.; Bruckner, R. J.; Paulo, J. A.; Cannon, J. R.; Ting, L.; Baltier, K.; Colby, G.; Gebreab, F.; Gygi, M. P.; Parzen, H.; Szpyt, J.; Tam, S.; Zarraga, G.; Pontano-Vaites, L.; Swarup, S.; White, A. E.; Schweppe, D. K.; Rad, R.; Erickson, B. K.; Obar, R. A.; Guruharsha, K. G.; Li, K.; Artavanis-Tsakonas, S.; Gygi, S. P.; Harper, J. W.
Architecture of the Human Interactome Defines Protein Communities and Disease Networks. Nature 2017, 545 (7655), 505-509.

(6) Milroy, L.-G.; Grossmann, T. N.; Hennig, S.; Brunsveld, L.; Ottmann, C. Modulators of Protein-Protein Interactions. Chem. Rev. 2014, 114 (9), 4695-4748.

(7) Arkin, M. R.; Tang, Y.; Wells, J. A. Small-Molecule Inhibitors of Protein-Protein Interactions: Progressing toward the Reality. Chem. Biol. 2014, 21 (9), 1102-1114.

(8) Andrei, S. A.; Sijbesma, E.; Hann, M.; Davis, J.; O’Mahony, G.; Perry, M. W. D.; Karawajczyk, A.; Eickhoff, J.; Brunsveld, L.; Doveston, R. G.; Milroy, L. G.; Ottmann, C. Stabilization of ProteinProtein Interactions in Drug Discovery. Expert Opin. Drug Discovery 2017, 12 (9), 925-940.

(9) Vogel, C.; Bashton, M.; Kerrison, N. D.; Chothia, C.; Teichmann, S. A. Structure, Function and Evolution of Multidomain Proteins. Curr. Opin. Struct. Biol. 2004, 14 (2), 208-216.

(10) Wu, H. Higher-Order Assemblies in a New Paradigm of Signal Transduction. Cell 2013, 153 (2), 287-292.

(11) den Hamer, A.; Rosier, B. J. H. M.; Brunsveld, L.; de Greef, T. F. A. Protein Scaffolds and Higher-Order Complexes in Synthetic Biology. In Synthetic Biology; Ryadnov, M., Brunsveld, L., Suga, H., Eds.; Royal Society of Chemistry: Cambridge, 2017; Vol. 2, pp 6596.

(12) Good, M. C.; Zalatan, J. G.; Lim, W. A. Scaffold Proteins: Hubs for Controlling the Flow of Cellular Information. Science 2011, 332 (6030), 680-686.

(13) Gordley, R. M.; Bugaj, L. J.; Lim, W. A. Modular Engineering of Cellular Signaling Proteins and Networks. Curr. Opin. Struct. Biol. 2016, 39, 106-114.

(14) Ottinger, E. A.; Botfield, M. C.; Shoelson, S. E. Tandem SH2 Domains Confer High Specificity in Tyrosine Kinase Signaling. J. Biol. Chem. 1998, 273 (2), 729-735.

(15) Ruthenburg, A. J.; Li, H.; Patel, D. J.; David Allis, C. Multivalent Engagement of Chromatin Modifications by Linked Binding Modules. Nat. Rev. Mol. Cell Biol. 2007, 8 (12), 983-994.

(16) Oh, D.; Ogiue-Ikeda, M.; Jadwin, J. A.; Machida, K.; Mayer, B. J.; Yu, J. Fast Rebinding Increases Dwell Time of Src Homology 2 (SH2)-Containing Proteins near the Plasma Membrane. Proc. Natl. Acad. Sci. U. S. A. 2012, 109 (35), 14024-14029.

(17) Wagner, M. J.; Stacey, M. M.; Liu, B. A.; Pawson, T. Molecular Mechanisms of SH2- and PTB-Domain-Containing Proteins in Receptor Tyrosine Kinase Signaling. Cold Spring Harbor Perspect. Biol. 2013, 5 (12), a008987-a008987.

(18) Csizmok, V.; Follis, A. V.; Kriwacki, R. W.; Forman-Kay, J. D. Dynamic Protein Interaction Networks and New Structural Paradigms in Signaling. Chem. Rev. 2016, 116 (11), 6424-6462.

(19) Kostrhon, S.; Kontaxis, G.; Kaufmann, T.; Schirghuber, E.; Kubicek, S.; Konrat, R.; Slade, D. A Histone-Mimicking Interdomain Linker in a Multidomain Protein Modulates Multivalent Histone Binding. J. Biol. Chem. 2017, 292 (43), 17643-17657.

(20) de Vink, P. J.; Briels, J. M.; Schrader, T.; Milroy, L.-G.; Brunsveld, L.; Ottmann, C. A Binary Bivalent Supramolecular Assembly Platform Based on Cucurbit[8]Uril and Dimeric Adapter Protein 14-3-3. Angew. Chem., Int. Ed. 2017, 56 (31), 8998-9002.

(21) Nyarko, A. Differential Binding Affinities and Allosteric Conformational Changes Underlie Interactions of Yorkie and a Multivalent PPxY Partner. Biochemistry 2018, 57 (5), 547-556.

(22) Mittag, T.; Orlicky, S.; Choy, W.-Y.; Tang, X.; Lin, H.; Sicheri, F.; Kay, L. E.; Tyers, M.; Forman-Kay, J. D. Dynamic Equilibrium Engagement of a Polyvalent Ligand with a Single-Site Receptor. Proc. Natl. Acad. Sci. U. S. A. 2008, 105 (46), 17772-17777.

(23) Tompa, P. Multisteric Regulation by Structural Disorder in Modular Signaling Proteins: An Extension of the Concept of Allostery. Chem. Rev. 2014, 114 (13), 6715-6732.

(24) Wright, P. E.; Dyson, H. J. Intrinsically Disordered Proteins in Cellular Signalling and Regulation. Nat. Rev. Mol. Cell Biol. 2015, 16 (1), 18-29.

(25) Mukhopadhyay, H.; de Wet, B.; Clemens, L.; Maini, P. K.; Allard, J.; van der Merwe, P. A.; Dushek, O. Multisite Phosphorylation 
Modulates the $\mathrm{T}$ Cell Receptor $\zeta$-Chain Potency but Not the Switchlike Response. Biophys. J. 2016, 110 (8), 1896-1906.

(26) Bah, A.; Forman-Kay, J. D. Modulation of Intrinsically Disordered Protein Function by Post-Translational Modifications. J. Biol. Chem. 2016, 291 (13), 6696-6705.

(27) Babu, M. M. The Contribution of Intrinsically Disordered Regions to Protein Function, Cellular Complexity, and Human Disease. Biochem. Soc. Trans. 2016, 44 (5), 1185-1200.

(28) Banjade, S.; Wu, Q.; Mittal, A.; Peeples, W. B.; Pappu, R. V.; Rosen, M. K. Conserved Interdomain Linker Promotes Phase Separation of the Multivalent Adaptor Protein Nck. Proc. Natl. Acad. Sci. U. S. A. 2015, 112 (47), E6426-E6435.

(29) Banani, S. F.; Lee, H. O.; Hyman, A. A.; Rosen, M. K. Biomolecular Condensates: Organizers of Cellular Biochemistry. Nat. Rev. Mol. Cell Biol. 2017, 18 (5), 285-298.

(30) Lin, Y.; Currie, S. L.; Rosen, M. K. Intrinsically Disordered Sequences Enable Modulation of Protein Phase Separation through Distributed Tyrosine Motifs. J. Biol. Chem. 2017, 292 (46), 1911019120.

(31) Eberhard, H.; Diezmann, F.; Seitz, O. DNA as a Molecular Ruler: Interrogation of a Tandem SH2 Domain with Self-Assembled, Bivalent DNA-Peptide Complexes. Angew. Chem., Int. Ed. 2011, 50 (18), 4146-4150.

(32) Scott, D. E.; Bayly, A. R.; Abell, C.; Skidmore, J. Small Molecules, Big Targets: Drug Discovery Faces the Protein-Protein Interaction Challenge. Nat. Rev. Drug Discovery 2016, 15 (8), 533550.

(33) Checco, J. W.; Gellman, S. H. Targeting Recognition Surfaces on Natural Proteins with Peptidic Foldamers. Curr. Opin. Struct. Biol. 2016, 39, 96-105.

(34) McIntyre, P. J.; Collins, P. M.; Vrzal, L.; Birchall, K.; Arnold, L. H.; Mpamhanga, C.; Coombs, P. J.; Burgess, S. G.; Richards, M. W.; Winter, A.; Veverka, V.; Delft, F. V.; Merritt, A.; Bayliss, R. Characterization of Three Druggable Hot-Spots in the Aurora-A/ TPX2 Interaction Using Biochemical, Biophysical, and FragmentBased Approaches. ACS Chem. Biol. 2017, 12 (11), 2906-2914.

(35) Aghazadeh, Y.; Papadopoulos, V. The Role of the 14-3-3 Protein Family in Health, Disease, and Drug Development. Drug Discovery Today 2015, 21 (2), 278-287.

(36) Milroy, L. G.; Brunsveld, L.; Ottmann, C. Stabilization and Inhibition of Protein-Protein Interactions: The 14-3-3 Case Study. ACS Chem. Biol. 2013, 8 (1), 27-35.

(37) Stevers, L. M.; Sijbesma, E.; Botta, M.; MacKintosh, C.; Obsil, T.; Landrieu, I.; Cau, Y.; Wilson, A. J.; Karawajczyk, A.; Eickhoff, J.; Davis, J.; Hann, M.; O’Mahony, G.; Doveston, R. G.; Brunsveld, L.; Ottmann, C. Modulators of 14-3-3 Protein-Protein Interactions. J. Med. Chem. 2018, 61 (9), 3755-3778.

(38) Stevers, L. M.; Lam, C. V.; Leysen, S. F. R.; Meijer, F. A.; van Scheppingen, D. S.; de Vries, R. M. J. M.; Carlile, G. W.; Milroy, L. G.; Thomas, D. Y.; Brunsveld, L.; Ottmann, C. Characterization and Small-Molecule Stabilization of the Multisite Tandem Binding between 14-3-3 and the R Domain of CFTR. Proc. Natl. Acad. Sci. U. S. A. 2016, 113 (9), E1152-E1161.

(39) Stevers, L. M.; de Vries, R. M. J. M.; Doveston, R. G.; Milroy, L. G.; Brunsveld, L.; Ottmann, C. Structural Interface between LRRK2 and 14-3-3 Protein. Biochem. J. 2017, 474 (7), 1273-1287.

(40) Cheng, S. H.; Gregory, R. J.; Marshall, J.; Paul, S.; Souza, D. W.; White, G. A.; O’Riordan, C. R.; Smith, A. E. Defective Intracellular Transport and Processing of CFTR Is the Molecular Basis of Most Cystic Fibrosis. Cell 1990, 63 (4), 827-834.

(41) Liang, X.; Da Paula, A. C.; Bozóky, Z.; Zhang, H.; Bertrand, C. a; Peters, K. W.; Forman-Kay, J. D.; Frizzell, R. A. PhosphorylationDependent 14-3-3 Protein Interactions Regulate CFTR Biogenesis. Mol. Biol. Cell 2012, 23 (6), 996-1009.

(42) Bozoky, Z.; Krzeminski, M.; Muhandiram, R.; Birtley, J. R.; AlZahrani, A.; Thomas, P. J.; Frizzell, R. A.; Ford, R. C.; Forman-Kay, J. D. Regulatory R Region of the CFTR Chloride Channel Is a Dynamic Integrator of Phospho-Dependent Intra-and Intermolecular Interactions. Proc. Natl. Acad. Sci. U. S. A. 2013, 110, E4427-36.
(43) Nichols, R. J.; Dzamko, N.; Morrice, N. a; Campbell, D. G.; Deak, M.; Ordureau, A.; Macartney, T.; Tong, Y.; Shen, J.; Prescott, A. R.; Alessi, D. R. 14-3-3 Binding to LRRK2 Is Disrupted by Multiple Parkinson's Disease-Associated Mutations and Regulates Cytoplasmic Localization. Biochem. J. 2010, 430 (3), 393-404.

(44) Yang, X.; Lee, W. H.; Sobott, F.; Papagrigoriou, E.; Robinson, C. V.; Gu, J.; Doyle, D. A.; Elkins, J. M. Structural Basis for Protein Protein Interactions in the 14-3-3 Protein Family. Proc. Natl. Acad. Sci. U. S. A. 2006, 103 (46), 17237-17242.

(45) Krishnamurthy, V. M.; Semetey, V.; Bracher, P. J.; Shen, N.; Whitesides, G. M. Dependence of Effective Molarity on Linker Length for an Intramolecular Protein-Ligand System. J. Am. Chem. Soc. 2007, 129 (5), 1312-1320.

(46) Movileanu, L.; Schiff, E. A. Entropy-Enthalpy Compensation of Biomolecular Systems in Aqueous Phase: A Dry Perspective. Monatsh. Chem. 2013, 144 (1), 59-65.

(47) Wu, G.; Olesińska, M.; Wu, Y.; Matak-Vinkovic, D.; Scherman, O. A. Mining 2:2 Complexes from 1:1 Stoichiometry: Formation of Cucurbit[8]Uril-Diarylviologen Quaternary Complexes Favored by Electron-Donating Substituents. J. Am. Chem. Soc. 2017, 139 (8), $3202-3208$

(48) van der Lee, R.; Buljan, M.; Lang, B.; Weatheritt, R. J.; Daughdrill, G. W.; Dunker, A. K.; Fuxreiter, M.; Gough, J.; Gsponer, J.; Jones, D. T.; Kim, P. M.; Kriwacki, R. W.; Oldfield, C. J.; Pappu, R. V.; Tompa, P.; Uversky, V. N.; Wright, P. E.; Babu, M. M. Classification of Intrinsically Disordered Regions and Proteins. Chem. Rev. 2014, 114 (13), 6589-6631.

(49) Boston, P. F.; Jackson, P.; Thompson, R. J. Human 14-3-3 Protein: Radioimmunoassay, Tissue Distribution, and Cerebrospinal Fluid Levels in Patients with Neurological Disorders. J. Neurochem. 1982, 38, 1475-1482.

(50) Muda, K.; Bertinetti, D.; Gesellchen, F.; Hermann, J. S.; von Zweydorf, F.; Geerlof, A.; Jacob, A.; Ueffing, M.; Gloeckner, C. J.; Herberg, F. W. Parkinson-Related LRRK2Mutation R1441C/G/H Impairs PKA Phosphorylation of LRRK2 and Disrupts Its Interaction with 14-3-3. Proc. Natl. Acad. Sci. U. S. A. 2014, 111 (1), E34-43.

(51) Hood, C. A.; Fuentes, G.; Patel, H.; Page, K.; Menakuru, M.; Park, J. H. Fast Conventional Fmoc Solid-Phase Peptide Synthesis with HCTU. J. Pept. Sci. 2008, 14 (1), 97-101.

(52) Albericio, F.; Bofill, J. M.; El-Faham, A.; Kates, S. A. Use of Onium Salt-Based Coupling Reagents in Peptide Synthesis. J. Org. Chem. 1998, 63 (26), 9678-9683.

(53) Kim, Y.-W.; Grossmann, T. N.; Verdine, G. L. Synthesis of AllHydrocarbon Stapled $\alpha$-Helical Peptides by Ring-Closing Olefin Metathesis. Nat. Protoc. 2011, 6 (6), 761-771. 